\title{
The Scenographic Worlds
}

\author{
Rudian Zekthi \\ I mondi scenografici \\ (Uno sguardo comparativo tra le poetiche di Kafka e di Calvino)
}

\section{Doi:10.5901/ajis.2014.v3n4p173}

\section{Abstract}

Institutions and the urban environment in the real world are not just instruments to allow the existence of society, but - as organizers of physical and mental space - they turn into constituting elements of individual existence in the world. In this respect, they unavoidably assume a scenographic nature. But while in theatre scenography completely replaces the characters' world, in the real world scenography does not replace - it only intermediates - the world for individuals in a society. In the realist novel the scenographic nature of institutions and the urban environment is represented just as in the real world: these intermediate between the characters and the world. With this intermediation they achieve the expansion of scenography, the negotiation of tension, the definition of the characters' fate within the events they are involved in. Whereas in Kafka's novel the scenographic nature of institutions and urban environment is like that of a theatre: as a replacement of the world. Hence the tension is constant, the expansion of scenography impossible, the characters' culpability a fact - this does not depend on the events in which they are involved, but on the positions imposed to them by the scenography. We also encounter in Calvino the same scenographic arrangement of the world as in Kafka. Only that Kafka's characters are resistant towards the fact that their interiority is preceded and dominated by the scenographic nature of the world, whereas Calvino's characters conform with this domination of the scenographic worlds, adapting as a premise of their existence the obtainment of spectacle. Jean Baudrillad in his Amérique shows us that the use of scenography as a replacement of, not an intermediation with, the world is possible in the real world, namely in the North American continent (as distinct from the European continent), and the effect is again the same: the acceptance of domination, where the members of this society are dominated by the world, this domination not being accompanied by resistance or dilemmas.

Keywords: scenography, distance, culpability, interiority, exteriority.

\section{La Scenografia Nella Vita Quotidiana e Nel Teatro}

II relazionarsi con il mondo (di natura esteriore all'esistenza umana, mai totalmente raccoglibile) ha costretto storicamente l'uomo a cercare di ammorbidire l'immane pressione del mondo sull'esistenza umana, di collocare ammortizzatori tra sé ed il mondo.

Questa ammortizzazione si verifica anche nell'interiorità: ricordi, immaginazioni, speranze, concetti, teorie ecc., ma a noi interessa soffermarci sui meccanismi di ammortizzazione che agiscono dall'esterno come le istituzioni, i riti, le usanze, l'urbanistica. Dunque, la casa, il quartiere, la città, lo stato, sono essenzialmente dei cerchi centripeti tendenti a fare da involucro tra l'uomo ed il potere inumano del mondo.

L'attenuarsi dell'effetto-mondo sull'uomo, secondo le tesi di Gaston Bachelard, non solo funge da compositore dello spazio umano, ma produce anche una distanza 'sopportabile' dal mondo, diminuendo o ritardando dirette conseguenze del mondo sull'uomo, sul suo destino e sui suoi scopi.

Questa opera umana predisposta ad assicurare anche all'esterno una distanza accettabile tra l'uomo ed il mondo la chiameremo "scenografia", nel senso che essa serve come lo spazio base in cui sono destinati a svilupparsi i progetti, le relazioni e le vite dei membri di una società.

Come tale essa sembra che rappresenti la fondamentale funzione del mondo in rapporto con la gente: garante della distanza in modo permanente e labile: permanenza e labilità che rende relativa sul piano del presente l'acuto intervento del mondo sui destini degli uomini.

Diamo questo primo significato alla parola scenografia, in quanto nel linguaggio teatrale assume anche un altro significato: è legata all'intenzione e alla progettualità delle vite umane, condiziona e segnala convenzionalmente (da fuori) quello che succede dentro i personaggi; quello che succede all'interno dei personaggi si avverte dal modo in cui la scenografia compone lo spazio, stabilisce le distanze ed accentua le posizioni. Ma questo secondo significato della scenografia teatrale, la scenografia urbanistica nella vita reale non lo assume. 
Perché? Perché la scenografia urbana tiene a distanza il mondo, come abbiamo detto, al punto che il mondo possa perdere il suo peso specifico e la sua irriducibilità, ma non lo sospende, non lo annienta del tutto. La presenza del mondo non smette di sentirsi, negli effetti continui di quello che succede nelle vite umane, e, penetrando attraverso questi ammortizzatori, in qualche modo, la presenza del mondo condiziona il miglioramento ed il rinnovamento della scenografia adoperata dagli uomini. Questo vale perché ogni nuovo elemento nella scenografia, ogni suo prolungamento costituisce uno sforzo di affrontare il mondo, che, nel migliore dei casi, conferma quello che conosciamo sul mondo: lì ci sono sempre luoghi nuovi, spazi nuovi per garantire le distanze tra le identità. Dunque il preesistente mondo è la condizione preliminare per la costruzione delle distanze.

Invece, la scenografia teatrale sostituisce del tutto il mondo. Al teatro non esiste altro mondo che quello suo, il mondo ha solo una possibilità di esistenza, quella della vita scenografica. E la scenografia è data in modo preliminare, essa non si allarga dall'esterno, ma dall'interno, nel senso dello spostamento e della ricomposizione continua sia delle posizioni che delle distanze. É per questo che la distanza è sempre tesa e in pericolo di eliminazione. In questo senso, la scenografia teatrale si trasforma in un'espressione esteriore del destino dei personaggi ed essendo esistita inizialmente come distanza e poi come sostitutrice del mondo, essa non rimane neutra ai destini dei personaggi, ma li precede e li include.

Inoltre, un'altra differenza importante tra la scenografia sociale del nostro mondo e la scenografia teatrale, consiste nel fatto che la scenografia della vita quotidiana, stando in mezzo, tra uomo ed mondo, non fuoriesce dalla propria natura di oggetto e come ogni oggetto si sottomette allo sforzo dell'uomo di considerarla come un prolungamento di sè. In questo senso, essa non comunica nulla all'uomo, anzi, riflette soltanto oppure assorbe l'esteriore luce di questo essere. Viceversa, la scenografia teatrale, essendo sfuggita allo status dell'intermediatore con il mondo, perché lo sostituisce interamente, paradossalmente, proprio come realtà esteriore all'uomo, perde la natura di oggetto e blocca la predisposizione umana di considerare la scenografia come un prolungamento dell'essere umano.

In analogia con quello che osservò Goethe quando notò la natura contradditoria del discorso nel teatro, dove grazie alla dizione recitata, dunque enfatica degli attori (per essere ascoltati da tutto il pubblico) si realizza l'effetto contrario, l'innaturalezza del teatro in rapporto con la vita, nello stesso modo, dimostrando con la sua esteriorità acuta (dunque con il fatto che oltre non c'è più nulla) la mancanza del mondo, la scenografia sociale dimostra l'impotenza dei personaggi di oltrepassarla (in quanto oltre c'è il nulla). Conseguentemente, la scenografia, assumendo come il mondo, il ruolo da avvolgente esterno e definitivo dei personaggi, diventa definitivamente incontrollabile dai personaggi. Perché, alla fine, cosa si può utilizzare come ammortizzatore tra la scenografia e i personaggi? Come un'esistenza del tutto esterna e non più umanizzata. $\mathrm{E}$ in più, come esistenza totalmente proiettata all'esterno e non più riducibile a umanità, essa acquista natura di oracolo, possedendo quello che l'essere umano cerca ma non può mai avere 0 , in altre parole, essa prevede quello che l'essere umano non ha potuto conoscere ed assumere.

\section{La Scenografia Nel Romanzo Realista}

Nel romanzo realista la scenografia si riproduce identica a come si è sempre realizzata nella storia umana: la casa, il paese, la città, la strada, il mercato, si danno come presenze intermedie tra l'uomo e il mondo, intermediazione che viene accettata dalla perenne possibilità di nuovi spazi e di nuove distanze: paesaggi, case città, viaggi. Di conseguenza, in questo romanzo i personaggi hanno una specie di autonomia rispetto alla presenza della scenografia quando si scontrano con essa.

Dunque, in questo romanzo, la scenografia ripresenta lo status di intermediario della scenografia nella vita quotidiana; e di conseguenza essa non condiziona le vite dei personaggi perché i loro destini sono un prodotto dell'intreccio tra le loro motivazioni interiori (al di qua della scenografia) e dei segnali esterni del mondo (aldilà della scenografia). A questo punto, la scenografia è, nel caso migliore, un prolungamento dei personaggi e agisce 0 in modo neutrale verso il loro destino oppure - in seguito a qualche incidente - in modo negativo.

Così, in questo tipo di romanzo, la scenografia non basta ad includere le vite umane, e di conseguenza la scenografia serve come giustificazione per le esitazioni, i malintesi e le scelte sbagliate, e inevitabilmente come ostacolo e danno per le vite umane. (Invece il mondo gioca il ruolo dell'arbitro morale tra l'uomo e la scenografia, senza avere un ruolo diretto nei confronti dei destini umani, pur riuscendo a comprendere in sè anche i loro fallimenti).

Questa relazione tra la scenografia e i personaggi nel romanzo realista è condizionata (diversamente dalla stessa relazione nel teatro) essenzialmente dall'intermediazione che la scenografia opera tra l'uomo ed il mondo. Come tale, la scenografia deve essere senza influenze oppure deve influenzare positivamente le vite umane, altrimenti il mondo consolerà da lontano gli uomini accidentati dalla scenografia. 


\section{La Scenografia Nella Poetica di Kafka}

Milan Kundera, nel suo saggio L'arte del romanzo, si esprime così: "Tutta la vita interiore di K. è assorbita dalla situazione in cui è intrappolato, e niente di quello che potrebbe andare al di là di questa situazione (i ricordi di K., le sue riflessioni metafisiche, le sue considerazioni sugli altri) ci viene mai rivelato. Per Proust, l'universo interiore dell'uomo costituiva un miracolo, un infinito che era motivo di continuo stupore. Ma non è questo che stupisce Kafka. Egli non si chiede quali siano le motivazioni interne che determinano il comportamento dell'uomo. La sua domanda è radicalmente diversa: quali possibilità ha ancora l'uomo in un mondo in cui le determinazioni esterne sono diventate così schiaccianti che i moventi interni non hanno più nessun peso? Che cosa sarebbe cambiato, infatti, nel destino e nel comportamento di K., se egli avesse avuto delle pulsioni omosessuali o una dolorosa storia d'amore alle spalle? Niente". ${ }^{1}$

Per la prima volta, nei romanzi di Kafka, la scenografia si usa con la stessa funzione che essa ha nel teatro, come unica possibilità dell'esistenza del mondo, di un mondo che non si sente più lontano ma nei destini dei personaggi.

Questa scenografia che non è più oltre il mondo, esercita la sua influenza diretta su quello che succede ai personaggi, condizionando il loro destino. I personaggi possono essere compresi in anticipo grazie alla forma che assume la scenografia in cui si sviluppa la loro esistenza e qualsiasi loro azione è destinata a confermare la scenografia.

Non solo "Una storia quotidiana", "Un medico di paese", Dialogo con l'ubriaco", in cui le aberrazioni spaziali sono semplicemente effetti di un mondo scenografico, ma anche "Nella colonia penale", "La condanna", e fino al "Catello" e a "L'America" la scenografia come surrogato del mondo assume anche l'autorità di rivelare preliminarmente il destino dei personaggi.

(Attenendoci alla brevità di questo lavoro, ci soffermermo sulla novella "La condanna", che inizia secondo la maniera realista, giacchè la Russia per Giorgio Bendemen fa parte del mondo esterno, invisibile, oltre la citta, oltre l'ufficio, l'impresa, il fidanzamento, che invece sono parte della scenografia. La conversazione con il padre, però, trasforma questa scenografia da intermediazione del mondo ad unica realtà esistente. L'effetto della trasformazione della stanza del padre da elemento scenografico di intermediazione con il mondo oltre la Russia in suo sostitutore viene confermato dal fatto che Giorgio chiama "commediante" il padre e lui lo accetta. E se, secondo il padre, la colpa più grande di Giorgio è che a lui era bastato conoscere se stesso, questa è essenzialmente la colpa di ogni personaggio del romanzo realista: nel quale, appunto, ogni personaggio, indebolendo la presenza del mondo attraverso la scenografia, e trasformando la scenografia in un prolungamento di se stesso, si trasforma nell'unica realtà esistente.

II fatto che questi personaggi rifiutano che il mondo diventi scenografia è una prova del loro carattere egocentrico. $E$ in quanto personaggio egocentrico (diabolico, viene chiamato da suo padre) è colpevole e viene condannato all'annegamento. E l'esecuzione volontaria della condanna da parte di Giorgio è una ammissione di colpa: in verità, il suo mondo è sempre stato scenografico, dalla stanza del padre al ponte non esiste il mondo in quanto tale ma solo in quanto scenografia, ed oltre il ponte, come ultimo elemento della scenografia, non c'è nulla; dunque la scenografia è il tutto e la peggiore condanna è essere allontanato da questo tutto).

E in Kafka, dopo questa novità di genere - dell'adattamento nel romanzo del mondo scenografico - si nota comunque, come reazione, la negazione del dominio ( della oppressione) dei personaggi ad opera della scenografia. Questo rifiuto è, dal punto di vista del contenuto, espressione del bisogno di resistenza dell'uomo verso la brutalità organizzativa del moderno, mentre dal punto di vista formale, è espressione dell'impossibilità della poetica di Kafka di staccarsi radicalmente dalle forme del romanzo precedente.

Come in quel romanzo, in cui la scenografia funge da intermediaria, anche nei romanzi di Kafka, questo rifiuto della scenografia è espressione dell'impossibilità della poetica di Kafka di staccarsi radicalmente dal romanzo passato.

Come in quel romanzo, in cui la scenografia funge da intermediaria, anche nei romanzi di Kafka la scenografia (oramai non intermediaria del mondo ma unica possibilità di esistenza del mondo) anche se non è tale è obbligata ad essere neutrale oppure dannosa.

Calvino annulla gli effetti prima accennati della scenografia. Mentre in Kafka era proprio questo carattere scenografico del mondo che condizionava il valore della distanza, nel romanzo realista la scenografia stava tra i personaggi ed il mondo ed è continuamente in movimento (principalmente in allargamento) confermando con la sua espansione la presenza inevitabile del mondo. Perché questa espansione introduce nello spazio umano territori che un attimo prima, invisibili ma familiari, erano parte del mondo.

Questi nuovi territori, anche se apparentemente servono da contenitori di altre informazioni ed aumentano la 
tensione, essenzialmente (in questo caso si può tradurre formalmente) sono diminutive della tensione, in quanto aumentano la possibilità per aumentare le distanze tra i personaggi. Sicuramente, il termine tensione ha un significato relativo, perché in ogni opera d'arte di finzione la tensione è l'ossigeno che tiene in vita i personaggi, ma qui si vuol dire dire che l'allargamento delle distanze dà ritmo alla tensione, la ritarda e la preannuncia, forse incrementa la sua intensità attraverso questo ritardo - dunque proprio quando la distanza si allarga, la tensione si appiattisce. Questa riduzione della tensione, anche se temporanea, ha una funzione formale: dà possibilità ai personaggi di conservare e rafforzare la loro identità, senza essere obbligati, per questo, a sforzarsi. Questa mancanza di sforzo, dall'altro lato, dà ai personaggi un'innocenza iniziale, perché fa sì che sul loro destino abbia maggiore importanza la fabula/ il sujet.

Nella poetica di Kafka, il carattere scenografico del mondo annulla questi effetti: la scenografia è il mondo stesso e non la sua rappresentazione, essa è data una volta per sempre, essa non rende possibile l'allargamento delle distanze ma solo un loro spostamento. Di conseguenza i personaggi perdono l'innocenza iniziale (in quanto si devono riconciliare con l'idea che la distanza non è un regalo auto-comprensibile e che richiede sforzo per essere acquisita), sono durante tutto il tempo di fronte a due alternative: o compensarla, acquisendo la distanza dagli altri (danneggiandoli), oppure unire le proprie distanze, sovrapponendole (danneggiandosi).

Ciò determina nella prosa di Kafka un fenomeno costante: uno dei personaggi è tutto il tempo distanziato dal gruppo degli altri, mentre questo gruppo misto soffre la sovrapposizione di tutti in un punto, in modo che non si superi la distanza con il protagonista, ma noi diremmo in modo che non si permetta che il fautore del male non scampi alla colpa. Per questo Joseph K. è colpevole: nelle condizioni di una scenografia data una volta per tutte, dunque di un'innocenza non inizialmente data, lui sarà sicuramente innocente. L'innocenza preliminare veniva garantita dalla distanza preliminare, la distanza preliminare è garantita da una combinazione di tutta la troupe dei personaggi in uno, - ma nelle condizioni in cui il mondo non è la dimora delle distanze, ma la distanza è l'unica presenza possibile del mondo (perché questo è un mondo scenografico) l'allargamento della distanza è una violenza verso il mondo in quanto tale e per una colpa del genere non c'è giustificazione: essa va punita. Dunque, come dice Kundera, è un modo dell'esistenza del mondo (scenografico) in quanto tale - tutta esterna, la quale spiega la possibilità delle azioni interne dei personaggi.

\section{La Poetica di Calvino: Scenografia ed Esaltazione}

Questo superamento schiacciante dell'intenzionalità dell'identità ad opera dell'intenzionalità del mondo trasformato in scenografia viene concepito dai personaggi di Kafka come del tutto inaccettabile ed essi lottano tutto il tempo per evitare ciò, considerandolo come la causa principale dei loro fallimenti e delle loro impossibilità. Si può mostrare come Calvino, partendo da Kafka, segna una nuova posizione. A nostro avviso (prendendo in prestito e usando la citazione di Kundera), in Calvino, l'identità è sì parte di un mondo comunque scenografico, ma l'Autore non lo concepisce come un'anomalia oppure una trappola, bensì come una lieta conferma della piccolezza dell'identità e della sua identificazione (grazie a questa piccolezza) con il mondo. Come è avviene ciò?

Dal momento che i personaggi si sentono come espressione locale dell'intenzionalità globale del mondo, essi concepiscono come piacevole il carattere esterno della loro interiorità (non lo concepiscono come uno sforzo, come invece i personaggi di Kafka). Attraverso l'identificazione della loro intenzionalità con l'intenzionalità del mondo, i personaggi di Calvino subiscono lo stesso effetto: il mondo si trasforma in scenografia.

Questa realizzazione del mondo scenografico rende inutile l'osservazione di quello che succede all'interno dei personaggi: il loro senso si trova espresso al di fuori di loro, diluito nel senso scenografico del mondo. Questa mancanza di necessità di valorizzare l'interiorità dei personaggi, questa comprensione del fatto di essere trasparenti perché nulla impedisce lo sguardo e l'orientamento degli altri, questa mancanza di pentimento che deriva dalla superficialità, sono tutte espressioni del carattere formale dei personaggi, ma sono nello stesso tempo effetto della conciliazione della minutezza dell'identità con la grandezza del mondo, oppure gli effetti dell'impossibilità di quello che è piccolo di tenere dentro di sè ogni cosa sotto la pressione incontrollabile del mondo tendente a giganteggiare oppure delle componenti delle sue strutture.

In questo senso, il lieto svuotamento dei personaggi di Calvino dal compito della vita interiore, si realizza in due modi. In primis, la spettacolarizzazione delle azioni esteriori è compensatrice e ripagante dell'indolenza delle azioni interne (secondo questo ragionamento per nulla ironico "alla fine perché serve intensità delle azioni interne se non per produrre intensità delle azioni esteriori? E se le azioni esteriori sono per natura - per emulazione del mondo - intense, anzi spettacolari, ecco che non servono affatto le azioni interiori"!) E, secondo questa legittimazione, viene accettato l'assottigliarsi del vivere interiore delle identità ne "Il visconte dimezzato", "Il cavaliere inesistente" (due dei romanzi della trilogia "I nostri antenati" e solo in "Giochi infiniti", solo uno dei racconti dell'opera "Le Cosmicomiche"). 
In secondo luogo, le azioni interiori dell'identità si presentano come realtà convenzionali che non rappresentano l'identità ma il mondo in cui si trova nella situazione contingente (ognuno in una situazione del genere, parte dello stesso mondo avrebbe le stesse sensazioni interiori, che sono senza valore nella funzione classica per distinguere le identità l'una dall'altra). Le identità possono sostituirsi ma solo per prolungare lo spettacolo causa-effetto, sempre lo stesso nelle stesse condizioni dello stesso mondo, perché l'esaltazione del potere di questo mondo, l'infinità della sua verità, sono la sola cosa che ha valore. $E$ quello che accade nell'interiorità dei personaggi è convenzionale, nel senso che esprime senza resistenza l'uso, in uno spazio vuoto oppure in un caso più 'morbido', del dogma fondamentale e invariabile del mondo. Così sucede ne "Il Barone rampante" e in tutti i racconti che rimangono ne "Le cosmicomiche".

\section{5. "L’America"}

Nelle opere d'arte, dunque, la scenografia si trova in due versioni: come intermediaria tra i personaggi ed il loro mondo (soprattutto nel romanzo realista) e come surrogato del mondo (nel teatro e nella poetica di Kafka e di Calvino). Nel nostro mondo, invece, è possibile che la scenografia come compositrice dello spazio, oltre che nel ruolo di intermediaria, si presenti anche come surrogato della presenza del mondo?

Possiamo solo accennare un principio di risposta, che possa servire come premessa per riflessioni future.

Partendo da "Le cosmicomiche", notiamo una somiglianza incredibile tra questi racconti e il testo, non di finzione, "L'America" (1985) di Jean Baudrillard. In questo testo, l'Autore presenta il continente come una presenza dal carattere acutamente esteriore, la cui essenza sta nel fatto che con lo spettacolo della propria esteriorità fa impallidire e rende convenzionale (attraverso il simulacro) il valore interiore di quel che succede nell'interiorità dei suoi abitanti, "gli americani".

La relativizzazione, anzi l'annullamento dell'interiorità umana può essere messa in atto dal giganteggiare della civiltà, dal peso infinito che esercitano i poteri molteplici delle grandi città, delle grandi distanze, delle grandi strade, dal superpotere che l'uomo vede incarnate nell'America come cultura e continente, come modo di vivere e come realtà di fatto.

Questo superpotere, queste megastrutture, questa civiltà-discoteca, in cui anche il rumore ed il silenzio, la luce ed il vuoto, sono estremi, comportano come effetto grandioso l'aumento della pressione sull'interiorità, al punto che, senza nemmeno rendersene conto, l'individuo non può tenere più dentro di sé il sentire, impoverisce la propria sensibilità, che si prosciuga lasciando l'individuo come una cavità, necessaria per moltiplicare l'eco di questo mondo.

E gli individui si riconciliano con questo vuoto, in primis in quanto espressione pacifica del loro prendere parte al mondo direttamente attraverso una civiltà scenografica, e in secondo luogo perché tutto quel che serve non è più necessario cercarlo nell'interiorità, ce n'è sempre un'incessante offerta fuori di sè.

(Ecco una citazione, che tra le altre lo suggerisce:

Libéré n'est pas l'homme dans sa réalité idéale, dans sa vérité intérieure ou dans sa transparence - libéré est l'homme qui change d'espace, qui circule, qui change de sexe, de vêtements, de mœurs selon la mode, et non selon la morale, qui change d'opinion selon les modèles d'opinion, et non selon sa conscience. C'est ça la libération pratique, qu'on le veuille ou non, qu'on en déplore ou non le gaspillage et l'obscénité. D'ailleurs, les gens des pays "totalitaires" savent bien que c'est lá la liberté vraie, ils ne rêvent que de cela: la mode, les modèles, les idoles, le jeu des images, pouvoir circuler pour circuler, la publicité, le déchaînement publicitaire. L'orgie, quoi. Or il faut bien dire que c'est l'Amérique qui a réalisé concrètement, techniquement cette orgie de libération, cette orgie de l'indifférence, de la déconnexion, de l'exhibition et de la circulation.) $)^{2}$

\section{Bibliografia}

Bachelard, Gaston- La poétique de l'espace, Paris, Presses Universitaires de France, 1957.

Baudrillard, Jean - Amerique Paris, Grasset 1986.

Kundera, Milan- L'arte del romanzo, Milano, Adelphi 1988.

\footnotetext{
2 Jean Baudrillard Amerique Paris, Grasset 1986,pp 94.
} 
